\title{
EFFECTS OF THYROID HORMONE ON GLYCATED HAEMOGLOBIN IN NON-DIABETIC SUBJECTS WITH OVERT HYPOTHYROIDISM
}

\author{
Mohammed Ismail ${ }^{1}$, Irfan Ahamed H. B2, Vithal Rao ${ }^{3}$, M. N. Lakshmikanth Reddy ${ }^{4}$, Syed Aman Jagirdar 5 \\ 1 Postgraduate Student, Department of Medicine, Al Ameen Medical College, Bijapur. \\ ${ }^{2}$ Assistant Professor, Department of Medicine, Al Ameen Medical College, Bijapur. \\ 3 Professor, Department of Medicine, Al Ameen Medical College, Bijapur. \\ ${ }_{4}^{4}$ Assistant Professor, Department of Medicine, Al Ameen Medical College, Bijapur. \\ 5 Postgraduate Student, Department of Medicine, Al Ameen Medical College, Bijapur.
}

\section{ABSTRACT}

\section{BACKGROUND}

HbA1c is an important parameter in diagnosis of diabetes. However, many other factors can independently influence HbA1c levels. The present study was aimed to determine the affect hypothyroidism on HbA1c level.

\section{MATERIALS AND METHODS}

50 individuals were selected for this study who attended Al-Ameen Medical College Hospital from December 2014 to October 2016 after applying inclusion and exclusion criteria. 25 served as cases and other 25 as control. Relevant investigations were carried out. Thyroid hormone replacement was initiated in 25 hypothyroid cases and they were again reviewed after 6 to 12 weeks. TSH and HbA1c along with other relevant investigations were repeated.

\section{RESULTS}

A total of 25 cases and 25 controls were studied with comparable mean age in both groups. The mean HbA1c in hypothyroid cases is $5.58 \%$ and in controls is $5.4 \%$, the difference between the means was mild but statistically not significant. The mean TSH in cases was 89.6 and in controls was 2.28 . The difference between the means was statistically significant $(p<0.001)$. All the 25 case s were evaluated again on second visit. There was a statistically significant reduction in mean TSH $(p=<0.001)$ and HbA1c $(p=0.033)$ after thyroid replacement therapy when compared to pretreatment values in cases.

\section{CONCLUSION}

HbA1c and TSH levels were found to be high in non-diabetic hypothyroid subjects compared normal controls and there was drop in levels of HbA1c and TSH after initiation of thyroid hormone replacement.

\section{KEYWORDS}

HbA1c, Hypothyroidism, TSH, Thyroid Hormone Replacement.

HOW TO CITE THIS ARTICLE: Ismail M, Ahamed IHB, Rao V, et al. Effects of thyroid hormone on glycated haemoglobin in nondiabetic subjects with overt hypothyroidism. J. Evolution Med. Dent. Sci. 2017;6(11):882-887, DOI: 10.14260/Jemds/2017/189

\section{BACKGROUND}

Reduced production of thyroid hormone is the central feature of the clinical state termed hypothyroidism.

Primary hypothyroidism which is due to disease of thyroid itself, accounts for approximately $99 \%$ of cases with less than $1 \%$ because of TSH deficiency and is termed central hypothyroidism.

Thyroid hormone playing an important role in the regulation of overall metabolic rate of the body has influence over the levels of glucose. The fluctuations in the values of glucose levels are reflected in the levels of HbA1c

Presently there is an increase in the number of studies on HbA1c. Several guidelines have been formulated on the role of HbA1c in the diagnosis of diabetes and its importance in labelling patients with high risk for diabetes, and its various

Financial or Other, Competing Interest: None.

Submission 16-12-2016, Peer Review 22-01-2017,

Acceptance 28-01-2017, Published 06-02-2017.

Corresponding Author:

Dr. Irfan Ahamed H. B,

S/o. K. B. Basheer Ahamed,

$1^{\text {st }}$ Stage, $2^{\text {nd }}$ Cross, Door \#15,

Iqbal Manzil, Dr. Ram Manohar Lohiya Nagar,

Shimoga - 577201, Karnataka.

E-mail:dr.irfan28@gmail.com

DOI: $10.14260 /$ jemds $/ 2017 / 189$ other "non-diabetic" uses. HbA1c was included as a diagnostic criterion first in the ADA guidelines of 2011 and since been a part of it. These guidelines were reviewed and refined in successive years. Since then the measurement of HbA1c by standard assays has retained its importance.

Several factors influence HbA1c among them glucose is the most important one affecting HbA1c. The type of assay used might also well influence the HbA1c value. Some haemoglobin traits, such as $\mathrm{HbS}, \mathrm{HbC}, \mathrm{HbF}$ and $\mathrm{HbE}$, interfere with some HbA1c assay methods. Haemolytic anaemia, blood transfusion, major blood loss, malaria etc. are the some of the conditions where in HbA1c results many not be obtained accurately. Apart from the aforementioned conditions some other morbidities like renal failure, iron deficiency anaemia, uraemia etc. are also speculated to influence HbA1c levels. However, one cannot be conclusive as further research is going on.

The influence of thyroid hormone on the levels of glycated haemoglobin and glycated albumin levels were studied and narrated by Kim et al and it was found that there was increased level of HbA1c in subjects with hypothyroidism compared to the control group. There was also decline in the levels of HbA1c following replacement of thyroid hormone. The above study is useful in this matter as the numbers of studies in this regard are sparse. [1] 
The aim of the present study is to investigate the levels of HbA1c in thyroid disorder namely hypothyroidism and also to investigate the influence of thyroid hormone replacement on the levels of HbA1c. The impetus for this study is the increasing prevalence of thyroid disorder and also to study its influence over the levels of HbA1c in overt hypothyroid and euthyroid groups.

\section{Thyroid Disorders and Diabetes Mellitus}

One of the most commonly encountered endocrine problem is diabetes mellitus. Due to the easy availability of assays to measure the thyroid hormones, this era has witnessed increase in the number of patients diagnosed to have subclinical or hypothyroidism. Diabetes and thyroid disorders have been shown to mutually influence each other and associations between both conditions have long been reported[2] Study by Coller and Huggins has shown that there was improvement of glucose tolerance in hyperthyroid patients having diabetes, after surgical resection of a parts of thyroid gland, hence proving the nascent relationship between hyperthyroidism and worsening glycaemia. [3] There is a deep underlying relation between diabetes mellitus and thyroid dysfunction. A plethora of studies have evidenced an array of complex intertwining biochemical, genetic, and hormonal malfunctions mirroring this pathophysiological association. [4]

\section{Incidence and Prevalence of Thyroid Disorders in the} General Population and in Patients with Diabetes

Thyroid disorders are fairly common with variable prevalence among the different population groups. In the Colorado Thyroid Disease Prevalence study involving 25,862 participants attending a state health fair, $9.5 \%$ of the studied population were found to have an elevated $\mathrm{TSH}$, while $2.2 \%$ had a low TSH.[5] Data from the Whickham survey conducted in the late $1970 \mathrm{~s}$ in the north of England revealed a prevalence of $6.6 \%$ of thyroid dysfunction in the adult general population. In the NHANES III study, a survey of 17,353 subjects representing the US population, hypothyroidism was found in $4.6 \%$ and hyperthyroidism in $1.3 \%$ of subjects. It was observed that an increased frequency of thyroid dysfunction with advancing age and a higher prevalence of thyroid disease in diabetic subjects compared to non-diabetic and women compared to men. Particularly, Perros et al. demonstrated an overall prevalence of $13.4 \%$ of thyroid diseases in diabetics with the highest prevalence of type 1 diabetes (31.4\%) in females and lowest prevalence in males with type 2 diabetes (6.9\%).[6] Autoimmunity has been implicated to be the major cause of thyroid-dysfunction associated diabetes mellitus. it was also seen that thyroid disorders were found to be more common in subjects with type 1 diabetes compared to those with type 2 diabetes possibly due to common autoimmune mechanism involved in pathogenesis of both. Additionally, a 3.5-fold increased risk of autoimmune thyroiditis was noticed in GADA positive patients.[7]

\section{Thyroid Hormones and Glucose Homeostasis}

Thyroid hormones affect glucose metabolism via several mechanisms. Hyperthyroidism has long been recognized to promote hyperglycaemia. ${ }^{[8]}$ A state of insulin resistance exists in patients with hyperthyroidism as shown by several studies. Hyperthyroidism has been associated with insulin resistance which has been linked with elevated glucose turnover, increased intestinal glucose absorption, elevated hepatic glucose output, increased free fatty acid concentrations, increased fasting and or postprandial insulin, increased peripheral glucose transport accompanied by glucose utilization. [9] Insulin resistance has been shown to be associated with subclinical hypothyroidism, which is in turn linked to impaired lipid balance and risk of development of metabolic syndrome. [10] During hyperthyroidism, the half-life of insulin is reduced most likely secondary to an increased rate of degradation and an enhanced release of biologically inactive insulin precursors. [11] Another mechanism explaining the relationship between hyperthyroidism and hyperglycaemia is the increase in glucose gut absorption mediated by the excess thyroid hormones. [12]

\section{Aims and Objectives}

- To study the effect of thyroid hormones on glycated haemoglobin in non-diabetics with overt hypothyroidism.

- To compare glycated haemoglobin in non-diabetic subjects with overt hypothyroidism and healthy euthyroid individuals.

- To study the effect of thyroid hormone replacement on glycated haemoglobin in non-diabetic hypothyroid patients, by comparing glycated haemoglobin pretreatment and 6-12 weeks after initiation of thyroid hormone replacement.

\section{MATERIALS AND METHODS}

\section{Source of the Data}

The study intends to include 50 patients (25 non-diabetic patients with overt hypothyroidism and 25 healthy euthyroid), attending OPD in Al Ameen medical college Hospital of the age group more than 18 and less than or equal to 50 years during the period from Dec 2014 - Oct 2016.

\section{Period of Study}

Dec. 2014-Oct. 2016.

\section{Design of Study}

Cross-sectional and Prospective Study.

\section{Methodology (Method of Collection of Data)}

50 patient study (25 non-diabetic patients with overt hypothyroidism and 25 healthy euthyroid), attending OPD in Al Ameen medical college Hospital of age group more than 18 and less than or equal to 50 years during the period from Dec 2014 - Oct 2016.

\section{Sample Selection}

Sample size $=50$

- 25 non-diabetic cases with newly diagnosed overt hypothyroidism.

- 25 non-diabetic euthyroid controls.

\section{Inclusion Criteria}

- Non-diabetics.

- Overt hypothyroidism (newly diagnosed).

- Age $>18$ years and Age $<50$ years. 


\section{Exclusion Criteria}

- Diabetes.

- Anaemia.

- Renal insufficiency.

- Liver dysfunction.

- Severe hypertriglyceridemia.

- Hypothyroid patients already on thyroid hormone replacement.

\section{Methods}

HbA1c levels were measured in 25 non-diabetic patients, with overt hypothyroidism and 25 healthy euthyroid controls.

HbA1c, Fasting Blood Sugar (FBS), and erythrocyte indices were determined in 25 non-diabetics with overt hypothyroidism, before and after hormone replacement.

Firstly, a cross sectional study was performed in 25 nondiabetic patients with newly diagnosed overt hypothyroidism. Patients with diabetes (ADA criteria), anaemia (haemoglobin< 10), renal insufficiency (serum creatinine> $1.5 \mathrm{mg} / \mathrm{dl}$ ), liver dysfunction and severe hypertriglyceridemia (triglycerides $>200 \mathrm{mg} / \mathrm{dl}$ ) were excluded.

For control subjects, 25 healthy euthyroid subjects were selected. Fasting blood samples were obtained for analysis of glucose, HbA1c, and thyroid stimulating hormone (TSH).

Secondly, a prospective trial was undertaken on nondiabetic patients with overt hypothyroidism. Patients were evaluated at the time of diagnosis of overt hypothyroidism (visit 1) and 6-12 weeks after subsequent thyroid hormone replacement (visit 2).

Patients with diabetes, anaemia, renal insufficiency, liver dysfunction and severe hypertriglyceridemia were already excluded. During visit 1, fasting blood samples were obtained from all patients for analysis of the haemoglobin level, mean corpuscular haemoglobin (MCH), mean corpuscular volume (MCV), TSH, free thyroxine (fT4), glucose (FBS), HbA1c. These variables were measured again during visit 2 .

The HbA1c level was measured using an automated high performance liquid chromatography analyser on BioRad D10. TSH level was measured using chemiluminescence immunoassay on centaur.

The paired Student $t$ test or the Wilcoxon signed rank test was used to compare variables before and after thyroid hormone replacement. Pearson correlation analysis was conducted to assess associations between variables. $\mathrm{P}<0.05$ was considered statistically significant.

\section{Statistical Analysis}

Mean and standard deviation (S.D.) was calculated for all the quantitative variables for in both cases and controls. Students unpaired $t$ test was used in comparison of two similar parameters in groups. Karl Pearson's correlation coefficient was calculated to correlate $\mathrm{HbA1c}$ and TSH in cases and controls. Calculations will be done using Microsoft excel and SPSS software.

\section{RESULTS}

Total of 25 non-diabetic individuals with hypothyroidism attending the outpatient clinic who satisfied the inclusion and exclusion criteria were taken up for the study along with 25 age and gender matched healthy individuals, who served as controls.

\begin{tabular}{|c|c|c|c|}
\hline & Cases & Control & P Value \\
\hline $\mathbf{N}$ & 25 & 25 & \\
\hline Male/Female & $7 \mathrm{M} / 18 \mathrm{~F}$ & $9 \mathrm{M} / 16 \mathrm{~F}$ & \\
\hline Age (yrs.) & $29.8 \pm 8.7$ & $30.4 \pm 8.5$ & 0.4 \\
\hline $\mathrm{FBS}(\mathrm{md} / \mathrm{dl})$ & $86.2 \pm 9.4$ & $78.5 \pm 8$ & 0.001 \\
\hline $\mathrm{HbA} 1 \mathrm{c} \%$ & $5.58 \pm 0.35$ & $5.4 \pm 0.3$ & 0.061 \\
\hline $\mathrm{TSH}(\mu \mathrm{IU} / \mathrm{ml})$ & $89.6 \pm 58.6$ & $2.28 \pm 1.32$ & $<0.001$ \\
\hline $\mathrm{Hb}(\mathrm{gm} \%)$ & $11.7 \pm 1.5$ & $12.56 \pm 1.74$ & 0.033 \\
\hline CREATININE $(\mathrm{mg} / \mathrm{dl})$ & $0.9 \pm 0.28$ & $0.76 \pm 0.16$ & 0.018 \\
\hline Table 1. The Mean of different variables in case and \\
control groups \\
\hline \multicolumn{4}{|c}{} \\
\hline
\end{tabular}

1. A total of 25 cases and 25 controls were studied in our study.

2. Cases included 7 male and 18 female and controls included 9 males and 16 females.

3. The mean age in cases is 29.8 years and in controls is 30.4 years and the difference between the groups was not significant.

4. The mean FBS in cases is $86.2 \mathrm{mg} / \mathrm{dl}$ and in controls is $78.5 \mathrm{mg} / \mathrm{dl}$, the difference between the means was statistically significant ( $\mathrm{p}=0.001)$.

5. The mean HbA1c in cases is $5.58 \%$ and in controls is $5.4 \%$, the difference between the means was statistically not significant $(\mathrm{p}=0.061)$.

6. The mean TSH in cases is $89.6 \mu \mathrm{IU} / \mathrm{ml}$ and in controls is $2.28 \mu \mathrm{IU} / \mathrm{ml}$, the difference between the means was statistically significant $(\mathrm{p}<0.001)$.

7. The mean $\mathrm{Hb}$ in cases is $11.7 \mathrm{gm} \%$ and in controls is $12.56 \mathrm{gm} \%$, the difference between the means was statistically significant ( $\mathrm{p}=0.033)$.

8. The mean Creatinine in cases is $0.9 \mathrm{mg} / \mathrm{dl}$ and in controls is $0.76 \mathrm{mg} / \mathrm{dl}$, the difference between the means was statistically significant ( $\mathrm{p}=0.018)$.

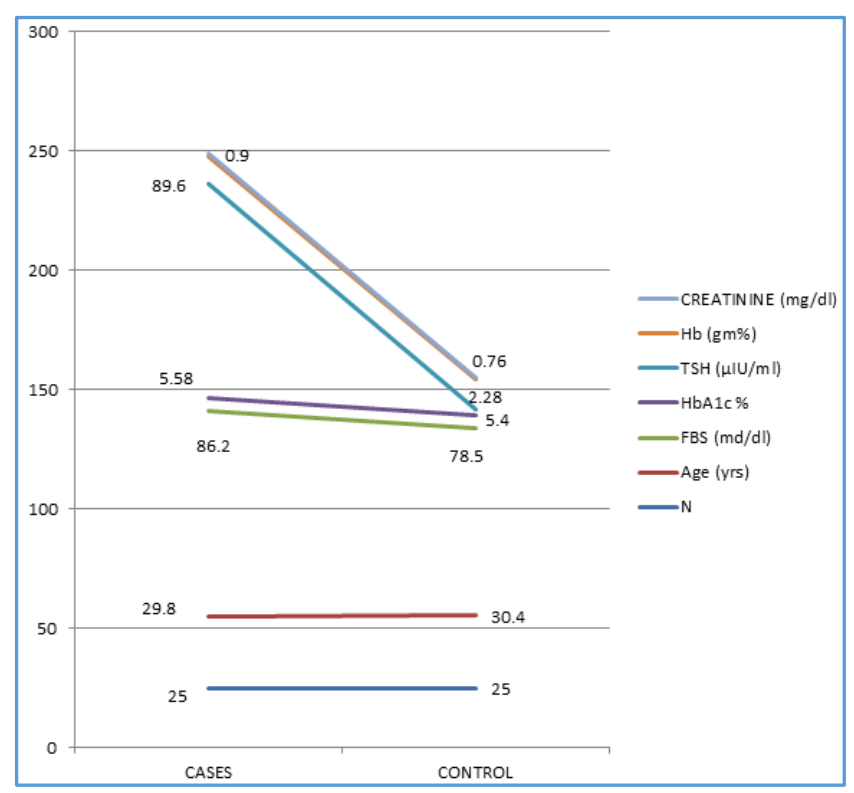

Chart 1. The Mean of different variables in case and control groups

The age of cases and controls ranged from 18 to 50 years. Out of the 25 cases, 7 were male and 18 were female. Out of the 25 controls, 9 were male and 16 were female. Most of the cases $(72 \%)$ and controls (64\%) were female. 
Pearson correlation coefficient used to analyse the relationship between TSH and HbA1c levels of cases was found to be very close to zero and the scatter diagram almost linear. Mild correlation, between TSH and HbA1c seen in our data.

Mean TSH in cases was 89.6 which was significantly higher than the TSH of 2.28 in the control group ( $p<0.001$ ) thus showing that hypothyroid individuals were used as cases and euthyroid individuals were selected as controls.

Mean $\mathrm{HbA1c}$ in cases was $5.58 \%$ and the mean HbA1c in controls was $5.44 \%$. Mean HbA1c in cases was found to be higher as compared to the controls suggesting a relatively higher HbA1c in hypothyroid individuals than in euthyroid individuals $(\mathrm{p}=0.061)$.

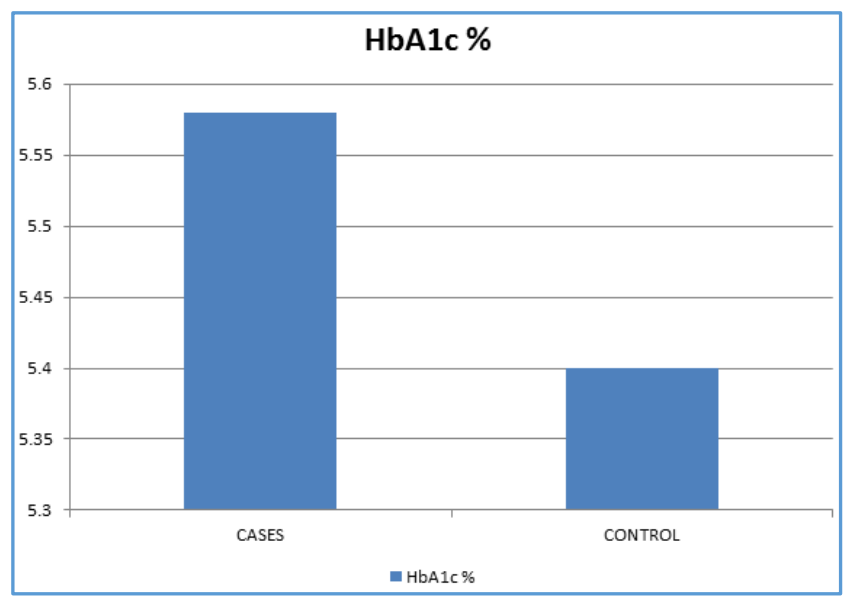

Chart 2. Bar Diagram depicting the difference between mean HbA1c levels in case group and control group Control Group HbA1c is lower than HbA1c in case group but the $p$ value was found to be 0.061 suggesting no statistical significance

The 25 cases were further evaluated for pre-treatment and post-treatment $\mathrm{HbA1c}$ levels after thyroid replacement therapy. The cases were requested for a follow-up visit at 6 to 12 weeks of initiation of therapy. On the second visit their HbA1c and TSH values were retaken and compared to the initial values taken prior to commencement of therapy.

\begin{tabular}{|c|c|c|c|}
\hline & $\begin{array}{c}\text { Pre } \\
\text { Treatment }\end{array}$ & $\begin{array}{c}\text { Post } \\
\text { Treatment }\end{array}$ & $\begin{array}{c}\text { P } \\
\text { value }\end{array}$ \\
\hline $\mathrm{TSH}(\mu \mathrm{IU} / \mathrm{ml})$ & 89.66 & 14.76 & $<0.001$ \\
\hline $\mathrm{HbA} 1 \mathrm{c} \%$ & 5.58 & 5.44 & 0.033 \\
\hline $\mathrm{HB} \mathrm{gm} \%$ & 11.7 & 12.4 & 0.066 \\
\hline \multicolumn{3}{|c|}{ Table 2. Showing the trend of TSH, HbA1c AND } \\
Hb in cases, pre and post treatment
\end{tabular}

1. There was a statistically significant reduction in mean TSH after thyroid replacement therapy when compared to pretreatment values $(\mathrm{p}=<0.001)$.

2. There was a statistically significant reduction in mean HbA1c after thyroid replacement therapy when compared to pretreatment values $(\mathrm{p}=0.033)$.

3. There was increase in mean $\mathrm{Hb}$ levels after thyroid replacement therapy when compared to pretreatment values, however the increase is not statistically significant $(\mathrm{p}=0.066)$.
4. Post thyroid replacement therapy cases showed a reduction in HbA1c levels. The mean HbA1c pretreatment was $5.58 \%$ compared to the post treatment $\mathrm{HbA1} \mathrm{c}$ of $5.44 \%$. The $\mathrm{p}$ value $=0.033$ suggests a statistical significance. This data indicated that as the TSH levels fall in an individual the HbA1c levels also decrease.

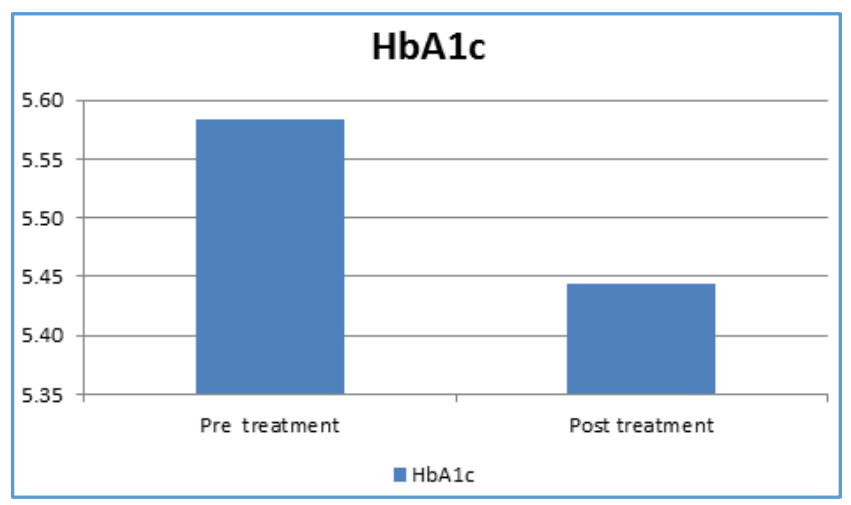

Chart 3. Bar Diagram depicting the difference between mean HbA1c levels pre-treatment and post treatment in cases

Post thyroid replacement therapy cases showed a reduction in HbA1c levels. The mean HbA1c pre-treatment was $5.58 \%$ compared to the post treatment $\mathrm{HbA} 1 \mathrm{c}$ of $5.44 \%$. The $\mathrm{p}$ value $=0.033$ suggests a statistical significance. This data indicated that as the TSH levels fall in an individual the HbA1c levels also decrease.

\section{DISCUSSION}

Hypothyroidism is commonly encountered endocrine disorder in present days.

This study was aimed to determine the effect of thyroid hormone on the levels of glycated haemoglobin in both deficient and replenished states. Hence it is important to study the effects of thyroid hormones on A1c, so as to help us in better interpretation of its levels in patients with thyroid dysfunction.

For the study 50 individuals were included, 25 of whom were non-diabetic hypothyroid individuals and the rest who were healthy euthyroid individuals serving as controls.

Individuals from 18 years to 50 years of age were included in the study, the age distribution of study cases was $52 \%$ from 18 to 28 years ( $\mathrm{n}=13), 36 \%$ from 29 to 39 years $(\mathrm{n}=9)$, and $12 \%$ from 40 to 50 years $(\mathrm{n}=3)$.

Keeping in mind the various other non-glycaemic factors affecting glycated haemoglobin, the inclusion and exclusion criterion were formulated. Some confounding medical conditions include acute and chronic blood loss, haemolytic anaemia, other anaemias, haemoglobin variants, blood urea and serum creatinine.[13]

\section{Prevalence}

The data suggests decreased prevalence of overt hypothyroidism as age increased, this is not concordant with the prevalence in the general population as observed by many studies showing an increased prevalence in the elderly. The Colorado thyroid disease prevalence study and the Wickham survey, one among the many studies demonstrated increased prevalence of hypothyroidism as age increases in the general population.[14] 


\section{Gender}

Percentage of females was $68 \%$, indicating an increased prevalence of overt hypothyroidism in females. According to Vanderpump MPJ the prevalence of spontaneous hypothyroidism is between $1 \%$ and $2 \%$, and it is more common in older woman and about ten times more common in woman than in men. [15]

\section{Correlation between the Levels of TSH and HbA1c}

TSH and HbA1c levels were measured in both cases and controls. There was found to be a mild positive correlation ( 0.08 ) between serum TSH and glycated haemoglobin in the 25 cases (Non-diabetic, newly diagnosed hypothyroid individuals) in the present study. Kim MK et al conducted a study to determine the effects of thyroid hormones on glycated haemoglobin and glycated albumin. The study by Kim MK et al showed a strong positive correlation between TSH and glycated haemoglobin in the 45 cases that were studied by them. A comparison was held between mean TSH and mean HbA1c levels. Both were found to be higher in cases when compared to the controls. The mean TSH in cases was $89.6 \mathrm{v} / \mathrm{s}$ controls $2.28(\mathrm{p}=0.001)$, and the mean $\mathrm{HbA1c}$ in cases was $5.58 \% \mathrm{v} / \mathrm{s}$ controls $5.44 \%(\mathrm{p}=0.061)$. The above observation infers that glycated haemoglobin levels are higher in hypothyroid individuals as compared to those of same age and sex matched euthyroid individuals, in accordance with previously reported studies.

\section{Effect of Thyroid Hormone Replacement}

After drawing blood for necessary investigations, the thyroid hormone replacement was initiated in the chosen 25 hypothyroid subjects. They were reviewed 6 to 12 weeks after initiation of therapy. Out of the 25 hypothyroid cases all reported for follow-up within the stipulated time and were subjected to relevant investigations.

Comparison was held between pretreatment and post treatment HbA1c and TSH levels. A significant decline was noted in the post treatment values. Mean TSH levels were significantly decreased after treatment $(\mathrm{p}<0.001)$ along with mean $\mathrm{HbA1c}$ levels, that were also significantly reduced $(\mathrm{p}<0.033)$. The above results suggest that with treatment of the hypothyroid state the HbA1c levels fall. The study results were similar to study conducted by Kim MK et al. ${ }^{1}$

The study results, are in cohesion with previous studies, and suggest that thyroid hormones exert influence over glycated haemoglobin values. Future studies with larger number of subjects are required to precisely quantitate the effect of thyroid hormones on HbA1c and also to determine the underlying mechanism which brings about the aforementioned results.

\section{CONCLUSION}

- The inferred results from the study of data suggest mildly positive correlation between TSH and HbA1c values of the cases studied.

- Cases included 7 male and 18 female and controls included 9 males and 16 females.

- The mean age in cases is 29.8 years and in controls is 30.4 years and the difference between the groups was not significant.
- The mean FBS in cases is $86.2 \mathrm{mg} / \mathrm{dl}$ and in controls is $78.5 \mathrm{mg} / \mathrm{dl}$, the difference between the means was statistically significant $(\mathrm{p}=0.001)$.

- The mean HbA1c in cases is $5.58 \%$ and in controls is $5.4 \%$, the difference between the means was statistically not significant $(\mathrm{p}=0.061)$.

- The mean TSH in cases is $89.6 \mu \mathrm{IU} / \mathrm{ml}$ and in controls is $2.28 \mu \mathrm{IU} / \mathrm{ml}$, the difference between the means was statistically significant $(\mathrm{p}<0.001)$.

- The mean $\mathrm{Hb}$ in cases is $11.7 \mathrm{gm} \%$ and in controls is $12.56 \mathrm{gm} \%$, the difference between the means was statistically significant ( $\mathrm{p}=0.033$ ).

- The mean Creatinine in cases is $0.9 \mathrm{mg} / \mathrm{dl}$ and in controls is $0.76 \mathrm{mg} / \mathrm{dl}$, the difference between the means was statistically significant $(\mathrm{p}=0.018)$.

- The mean HbA1c level compared between cases (hypothyroid and non-diabetics) and controls (healthy, non-diabetic euthyroid individuals) was found to be lower in studied controls. ( $\mathrm{p}$ value $=0.061$ )

- A statistically significant difference in both TSH (p value $<0.001$ ) and HbA1c ( $p$ value $<0.033$ ) was established in pre-treatment and post-treatment values inferring that correction of hypothyroid states was correlated with fall in HbA1c levels.

- Hence, we conclude that thyroid hormone affects HbA1c values. HbA1c was found to be decreased after treatment and mean HbA1c was lower in euthyroid patients.

\section{REFERENCES}

[1] Kim MK, Kwon HS, Baek KH, et al. Effects of thyroid hormone on $\mathrm{A} 1 \mathrm{C}$ and glycated albumin levels in nondiabetic subjects with overt hypothyroidism. Diabetes Care 2010;33(12):2546-8.

[2] Feely J, Isles TE. Screening for thyroid dysfunction in diabetics. British Medical Journal 1979;1(6179):1678.

[3] Wang C. The relationship between type 2 diabetes mellitus and related thyroid diseases. Journal of Diabetes Research 2013;2013:390534.

[4] Brenta G, Danzi S, Klein I. Potential therapeutic applications of thyroid hormone analogs. Nat Clin Pract Endocrinol Metab 2007;3(9):632-40.

[5] Canaris GJ, Manowitz NR, Mayor G, et al. The colorado thyroid disease prevalence study. Arch Inter Med 2000;160(4):526-34.

[6] Perros P, McCrimmon RJ, Shaw G, et al. Frequency of thyroid dysfunction in diabetic patients: value of annual screening. Diabet Med 1995;12(7):622-7.

[7] Kordonouri 0, Charpentier N, Hartmann R. GADA positivity at onset of type 1 diabetes is a risk factor for the development of autoimmune thyroiditis. Pediatric Diabetes 2011;12(1):31-3.

[8] Maxon HR, Kreines KW, Goldsmith RE, et al. Long-term observations of glucose tolerance in thyrotoxic patients. Arch Intern Med 1975;135(11):1477-80.

[9] Brenta G, Celi FS, Pisarev M, et al. Acute thyroid hormone withdrawal in athyreotic patients results in a state of insulin resistance. Thyroid 2009;19(6):665-9.

[10] Rezzonico J, Niepomniszcze H, Rezzonico M, et al. The association of insulin resistance with subclinical thyrotoxicosis. Thyroid 2011;21(9):945-9. 
[11] O'Meara NM, Blackman JD, Sturis J, et al. Alterations in the kinetics of C-peptide and insulin secretion in hyperthyroidism. J Clin Endocrinol Metab 1993;76(1):79-84.

[12] Levin RJ, Smyth DH. The effect of the thyroid gland on intestinal absorption of hexoses. J Physiol 1963;169:755-69.

[13] Bernstein RE. Glycosylated hemoglobins: hematologic considerations determine which assay for glycohemoglobin is advisable. Clin Chem 1980;26(1):174-5.

[14] Tunbridge WM, Evered DC, Hall R. The spectrum of thyroid disease in a community: the whickham survey. Clinical Endocrinology 1977;7(6):481-93.

[15] Vanderpump MP, Turnbridge WM. Epidemiology and prevention of clinical and subclinical hypothyroidism. Thyroid 2002;12(10):839-47. 